\title{
Data report: calcium carbonate and total organic carbon records from IODP Expedition 339 Sites U1385 and U1391'
}

Fatima Abrantes $2,3,4$

\section{Chapter contents}

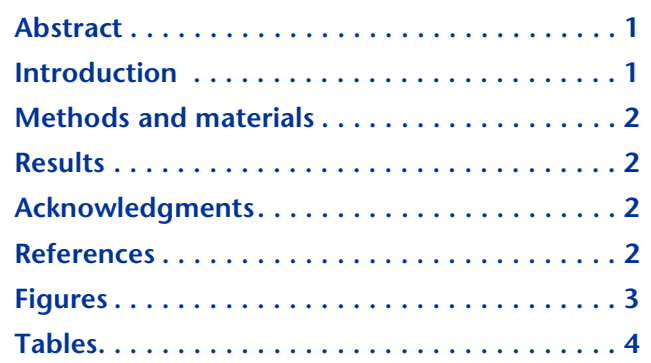

${ }^{1}$ Abrantes, F., 2016. Data report: calcium carbonate and total organic carbon records from IODP Expedition 339 Sites U1395 and U1391. In Stow, D.A.V., Hernández-Molina, F.J., Alvarez Zarikian, C.A., and the Expedition 339 Scientists, Proceedings of the Integrated Ocean Drilling Program, 339: Tokyo (Integrated Ocean Drilling Program Management International, Inc.). doi:10.2204/iodp.proc.339.201.2016 2Divisão de Geologia e Georecursos Marinhos, Instituto Português do Mar e da Atmosfera (IPMA), Rua Alfredo Magalhães Ramalho 6, 1495-006 Lisboa, Portugal. fatima.abrantes@ipma.pt ${ }^{3} \mathrm{Also}$ at Interdisciplinary Centre of Marine and Environmental Research (CIIMAR), Universidade do Porto, Rua dos Bragas 287, 4050-123 Porto, Portugal.

${ }^{4}$ Also at Centro de Ciencias do Mar (CCMAR), Universidade do Algarve, Campus de Gambelas, 8005-139 Faro, Portugal.

\begin{abstract}
Sedimentary inorganic calcium carbonate $\mathrm{CaCO}_{3}$ and total organic carbon (TOC) are two of the most commonly used indicators of past oceanic productivity. In this report, we present $\mathrm{CaCO}_{3}$ and TOC records from Integrated Ocean Drilling Program Expedition 339 Sites U1385 and U1391 drilled in the southwest Iberian margin. Identified as a key region to establish the marine-ice-terrestrial linkages crucial for understanding the Earth's climate system, this margin is also characterized by seasonal coastal upwelling-related high primary production. These IODP sites constitute a depth transect across a productivity gradient allowing for the investigation of the impact of climate variations on marine production and preservation.

A total of 420 samples were analyzed for the 75-100 meters composite depth (mcd) interval at Site U1385 and for the 160-220 mcd interval at Site U1391. Results show at least two scales of variability for both $\mathrm{CaCO}_{3}$ and TOC, which are comparable between sites but have a better-defined long-term trend on the Ca$\mathrm{CO}_{3}$ record.
\end{abstract}

\section{Introduction}

Sedimentary inorganic $\mathrm{CaCO}_{3}$ and total organic carbon (TOC) content in ocean sediments are qualitative indicators of past changes in marine production, which in turn is linked to the global carbon cycle. When coupled with isotopic data and other independent productivity proxies, they provide important information for understanding how marine ecosystems responded to past climate variability (Sarmiento and Gruber, 2006).

The Iberian margin is a coastal upwelling region where productivity increases in response to coastal upwelling generated by wind intensity and direction, and although upwelling centers are mainly coastal, upwelled water spreads offshore through filaments that can extend as far as $200 \mathrm{~km}$ offshore depending on the intensity of the northerly winds (Fiúza, 1983; Relvas et al., 2009). Sites U1385 and U1391 are located in the area influenced by the Cape Sines and Cape St. Vicente filaments (Sousa and Bricaud, 1992). The study of productivity proxies in these sedimentary sequences will provide information on past productivity and upwelling intensity. This report provides data on sedimentary 
$\mathrm{CaCO}_{3}$ and TOC measured at Sites U1385 (75-100 meters composite depth [mcd]) and U1391 (160-220 mcd).

\section{Methods and materials}

Sites U1385 $\left(37^{\circ} 34.285^{\prime} \mathrm{N}, 10^{\circ} 7.562^{\prime} \mathrm{W} ; 2578\right.$ meters below sea level [mbsl]) and U1391 $\left(37^{\circ} 21.5322^{\prime} \mathrm{N}\right.$, $9^{\circ} 24.6558^{\prime} \mathrm{W}$; $1080 \mathrm{mbsl}$ ) were drilled on the West Iberian margin as part of Integrated Ocean Drilling Program Expedition 339 (see the "Expedition 339 summary" chapter [Expedition 339 Scientists, 2013]). $\mathrm{CaCO}_{3}$ and TOC contents were determined for a total of 195 levels between 75 and 100 mcd at Site U1385 and 225 levels between 160 and $220 \mathrm{mcd}$ at Site U1391 (Tables T1, T2).

The $2 \mathrm{~cm}^{3}$ sediment samples from each level were dried and then powdered and homogenized on a Fritsch Pulverizette 7 rotary mortar. Three replicates of $2 \mathrm{mg}$ subsamples per level were then analyzed for total carbon using a CHNS-932 LECO elemental analyzer. The same set of samples was later subject to combustion for $8 \mathrm{~h}$ through a predefined stepwise increase in temperature up to $400^{\circ} \mathrm{C}$ and reanalyzed for inorganic carbon. The organic carbon content was determined by the difference between total carbon and inorganic carbon (TOC $=\mathrm{TC}-\mathrm{IC})$. Data are presented in weight percent. The relative precision of repeated measurements of both samples and standards was $0.03 \mathrm{wt} \%$. Analyses were done in the sedimentology and micropaleontology laboratory of the Marine Geology Division at the Portuguese Institute for the Ocean and Atmosphere (IPMA).

\section{Results}

Results of sedimentary inorganic and total organic carbon from Sites U1385 (75-100 mcd) and U1391 (160-220 mcd) are compiled in Tables T1 and T2 and presented in Figures F1 and F2.

$\mathrm{CaCO}_{3}$ values vary between 10 and $50 \mathrm{wt} \%$, whereas TOC values oscillate between 0.5 and $2 \mathrm{wt} \%$ along the analyzed intervals at both sites. A visual evaluation of the records reveals two levels of fluctuations in both TOC and $\mathrm{CaCO}_{3}$, also at both locations. However, at the longer wave variability, $\mathrm{CaCO}_{3}$ con- tents show larger amplitude of variation than TOC (Figs. F1, F2).

\section{Acknowledgments}

This research used samples provided by the Integrated Ocean Drilling Program (IODP). Funding was provided by FCT project PTDC/AAG-GLO/3737/ 2012, executed by Interdisciplinary Centre of Marine and Environmental Research (CIIMAR) at the National Laboratory for Energy and Geology (LNEG) and Portuguese Institute for the Ocean and the Atmosphere (IPMA). The author acknowledges Cremilde Monteiro and Daniel Ferreira for their laboratory support as well as Walter Hale and all the colleagues that participated on the sampling party and contributed to sampling of these sites.

\section{References}

Expedition 339 Scientists, 2013. Expedition 339 summary. In Stow, D.A.V., Hernández-Molina, F.J., Alvarez Zarikian, C.A., and the Expedition 339 Scientists, Proceedings of the Integrated Ocean Drilling Program, 339: Tokyo (Integrated Ocean Drilling Program Management International, Inc.). http://dx.doi.org/10.2204/ iodp.proc.339.101.2013

Fiúza, A., 1983. Upwelling patterns off Portugal. In Suess, E., and Thiede, J. (Eds.), NATO Conference Series (Volume 10B): Coastal Upwelling: Its Sediment Record (Part A): New York (Plenum), 85-98. http://dx.doi.org/10.1007/9781-4615-6651-9_5

Relvas, P., Luís, J., and Santos, A.M.P., 2009. Importance of the mesoscale in the decadal changes observed in the northern Canary upwelling system. Geophysical Research Letters, 36(22):L22601. http://dx.doi.org/10.1029/ 2009GL040504

Sarmiento, J.L., and Gruber, N., 2006. Ocean Biogeochemical Dynamics: Princeton, NJ (Princeton University Press).

Sousa, F.M., and Bricaud, A., 1992. Satellite-derived phytoplankton pigment structures in the Portuguese upwelling area. Journal of Geophysical Research: Oceans, 97(C7):11343-11356. http://dx.doi.org/10.1029/ 92JC00786

Initial receipt: 12 February 2015

Acceptance: 19 April 2016

Publication: 27 October 2016

MS 339-201 
Figure F1. Calcium carbonate and total organic carbon (TOC) from 75 to 100 mcd, Site U1385.

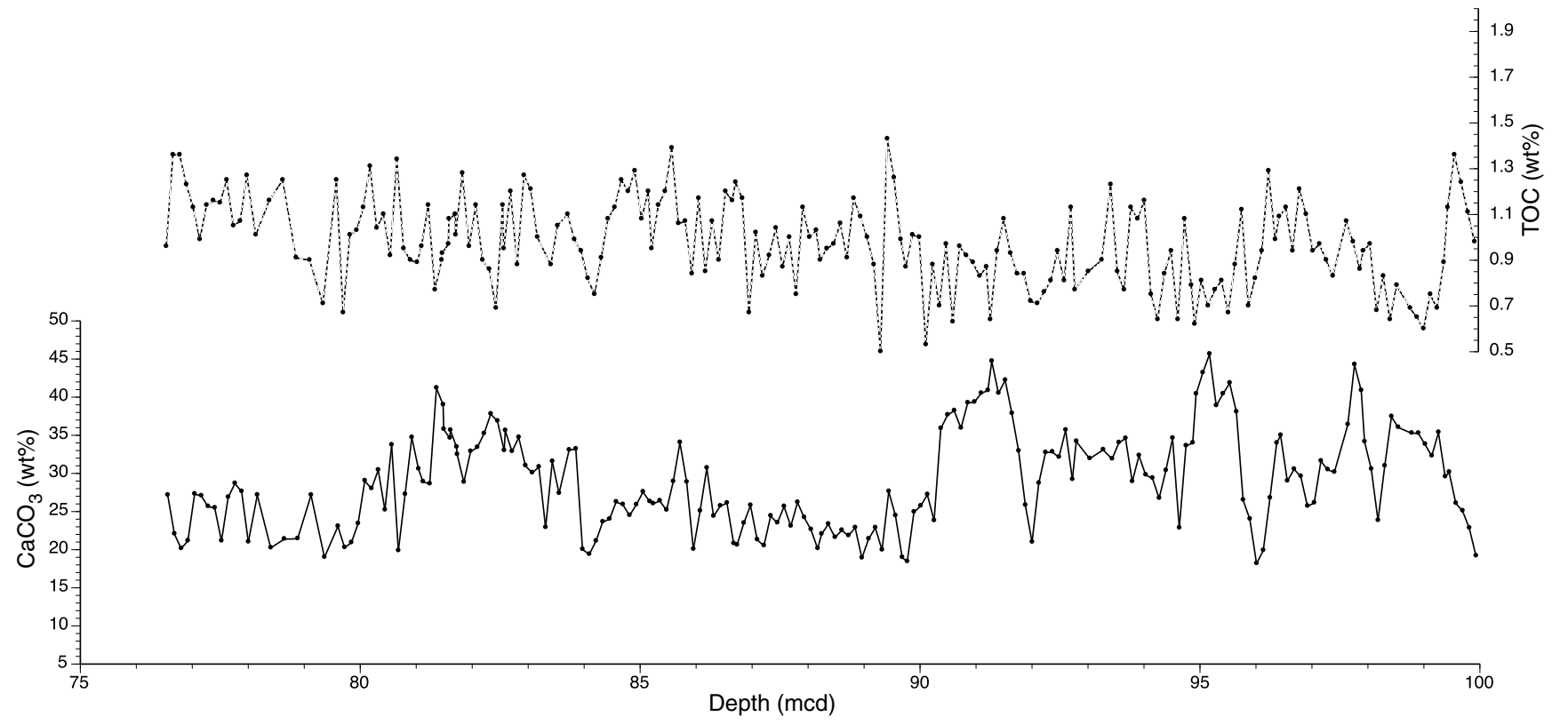

Figure F2. Calcium carbonate and total organic carbon (TOC) records from 160 to 220 mcd, Site U1391.

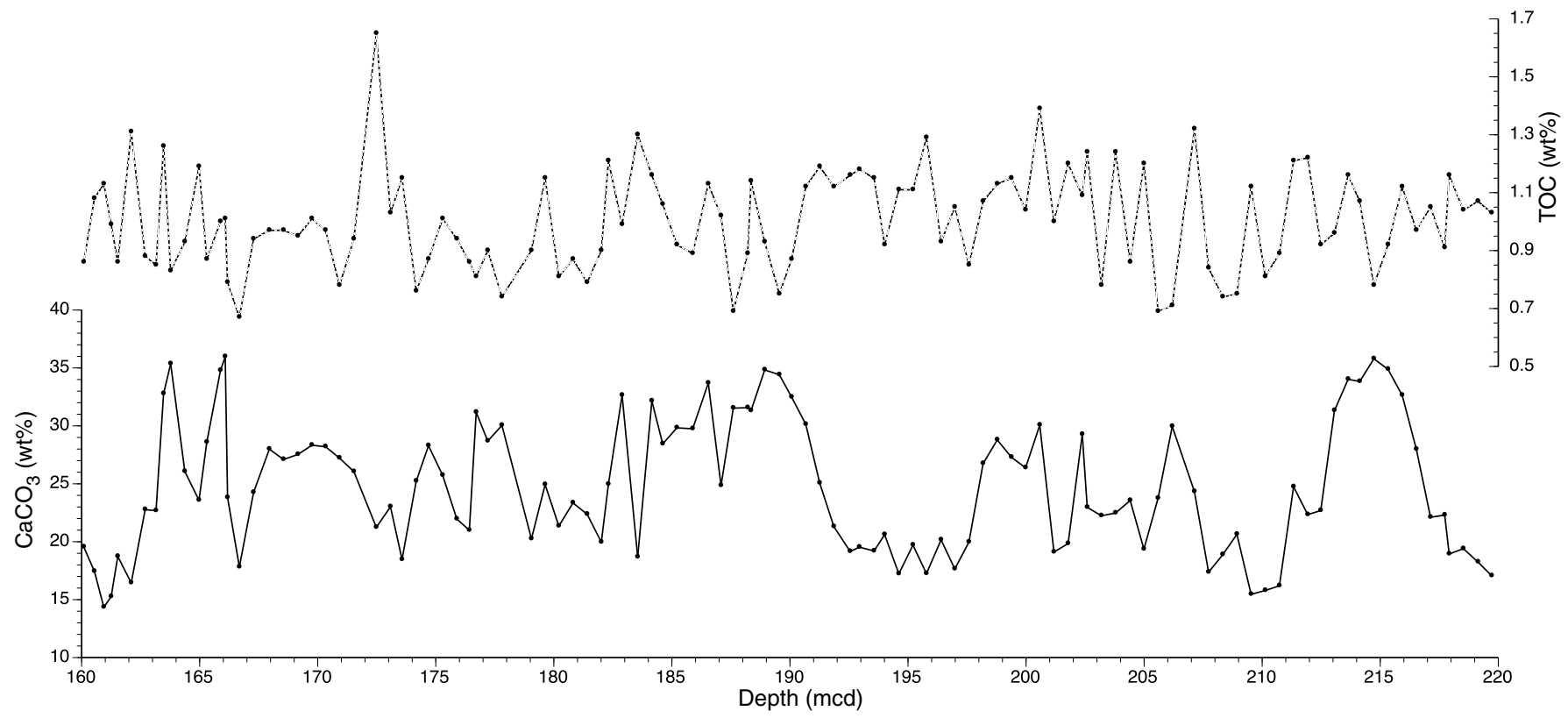


Table T1. Calcium carbonate and total organic carbon (TOC) concentrations from 75 to 100 mcd, Site U1385. (Continued on next page.)

\begin{tabular}{|c|c|c|c|c|}
\hline $\begin{array}{l}\text { Hole, core, section, } \\
\text { interval }(\mathrm{cm})\end{array}$ & $\begin{array}{c}\text { Top depth } \\
\text { (mbsf) }\end{array}$ & $\begin{array}{l}\text { Top depth } \\
\text { (mcd) }\end{array}$ & $\begin{array}{c}\text { TOC } \\
\text { (wt\%) }\end{array}$ & $\begin{array}{l}\mathrm{CaCO}_{3} \\
\text { (wt\%) }\end{array}$ \\
\hline \multicolumn{5}{|l|}{$339-$} \\
\hline U1385D-9H-3, 1-2 & 73.45 & 76.56 & 0.96 & 27.14 \\
\hline U1385D-9H-3, 13-14 & 73.57 & 76.68 & 1.36 & 22.05 \\
\hline U1385D-9H-3, 25-26 & 73.69 & 76.80 & 1.36 & 20.14 \\
\hline U1385D-9H-3, 37-38 & 73.81 & 76.92 & 1.23 & 21.15 \\
\hline U1385D-9H-3, 49-50 & 73.93 & 77.04 & 1.13 & 27.29 \\
\hline U1385D-9H-3, 61-62 & 74.05 & 77.16 & 0.99 & 27.05 \\
\hline U1385D-9H-3, 73-74 & 74.17 & 77.28 & 1.14 & 25.65 \\
\hline U1385D-9H-3, 85-86 & 74.29 & 77.40 & 1.16 & 25.46 \\
\hline U1385D-9H-3, 97-98 & 74.41 & 77.52 & 1.15 & 21.14 \\
\hline U1385D-9H-3, 109-110 & 74.53 & 77.64 & 1.25 & 26.86 \\
\hline U1385D-9H-3, 121-122 & 74.65 & 77.76 & 1.05 & 28.67 \\
\hline U1385D-9H-3, 133-134 & 74.77 & 77.88 & 1.07 & 27.62 \\
\hline U1385D-9H-3, 145-146 & 74.89 & 78.00 & 1.27 & 21.00 \\
\hline U1385D-9H-4, 7-8 & 75.05 & 78.16 & 1.01 & 27.15 \\
\hline U1385D-9H-4, 31-32 & 75.29 & 78.40 & 1.16 & 20.23 \\
\hline U1385D-9H-4, 55-56 & 75.53 & 78.64 & 1.25 & 21.38 \\
\hline U1385D-9H-4, 79-80 & 75.77 & 78.88 & 0.91 & 21.43 \\
\hline U1385D-9H-4, 103-104 & 76.01 & 79.12 & 0.90 & 27.14 \\
\hline U1385D-9H-4, 127-128 & 76.25 & 79.36 & 0.71 & 18.99 \\
\hline U1385D-9H-5, 1-2 & 76.49 & 79.60 & 1.25 & 23.05 \\
\hline U1385D-9H-5, 13-14 & 76.61 & 79.72 & 0.67 & 20.27 \\
\hline U1385D-9H-5, 25-26 & 76.73 & 79.84 & 1.01 & 20.91 \\
\hline U1385D-9H-5, 37-38 & 76.85 & 79.96 & 1.03 & 23.41 \\
\hline U1385D-9H-5, 49-50 & 76.97 & 80.08 & 1.13 & 29.02 \\
\hline U1385D-9H-5, 61-62 & 77.09 & 80.20 & 1.31 & 28.00 \\
\hline U1385D-9H-5, 73-74 & 77.21 & 80.32 & 1.04 & 30.43 \\
\hline U1385D-9H-5, 85-86 & 77.33 & 80.44 & 1.10 & 25.20 \\
\hline U1385D-9H-5, 97-98 & 77.45 & 80.56 & 0.92 & 33.72 \\
\hline U1385D-9H-5, 109-110 & 77.57 & 80.68 & 1.34 & 19.86 \\
\hline U1385D-9H-5, 121-122 & 77.69 & 80.80 & 0.95 & 27.25 \\
\hline U1385D-9H-5, 133-134 & 77.81 & 80.92 & 0.90 & 34.70 \\
\hline U1385D-9H-5, 145-146 & 77.93 & 81.04 & 0.89 & 30.57 \\
\hline U1385D-9H-6, 1-2 & 78.01 & 81.12 & 0.96 & 28.90 \\
\hline U1385D-9H-6, 13-14 & 78.13 & 81.24 & 1.14 & 28.62 \\
\hline U1385D-9H-6, 25-26 & 78.25 & 81.36 & 0.77 & 41.19 \\
\hline U1385D-9H-6, 37-38 & 78.37 & 81.48 & 0.90 & 38.99 \\
\hline U1385E-9H-6, 37-38 & 72.20 & 81.49 & 0.93 & 35.77 \\
\hline U1385D-9H-6, 49-50 & 78.49 & 81.60 & 0.97 & 34.62 \\
\hline U1385E-9H-6, 49-50 & 72.32 & 81.61 & 1.08 & 35.65 \\
\hline U1385D-9H-6, 61-62 & 78.61 & 81.72 & 1.10 & 33.44 \\
\hline U1385E-9H-6, 61-62 & 72.44 & 81.73 & 1.01 & 32.48 \\
\hline U1385E-9H-6, 73-74 & 72.56 & 81.85 & 1.28 & 28.84 \\
\hline U1385E-9H-6, 85-86 & 72.68 & 81.97 & 0.96 & 32.87 \\
\hline U1385E-9H-6, 97-98 & 72.80 & 82.09 & 1.14 & 33.40 \\
\hline U1385E-9H-6, 109-110 & 72.92 & 82.21 & 0.90 & 35.21 \\
\hline U1385E-9H-6, 121-122 & 73.04 & 82.33 & 0.86 & 37.78 \\
\hline U1385E-9H-6, 133-134 & 73.16 & 82.45 & 0.69 & 36.86 \\
\hline U1385E-9H-6, 145-146 & 73.28 & 82.57 & 1.14 & 32.99 \\
\hline U1385E-9H-7, 1-2 & 73.30 & 82.59 & 0.95 & 35.61 \\
\hline U1385E-9H-7, 13-14 & 73.42 & 82.71 & 1.20 & 32.87 \\
\hline U1385E-9H-7, 25-26 & 73.54 & 82.83 & 0.88 & 34.71 \\
\hline U1385E-9H-7, 37-38 & 73.66 & 82.95 & 1.27 & 31.00 \\
\hline U1385E-9H-7, 49-50 & 73.78 & 83.07 & 1.21 & 30.07 \\
\hline U1385E-9H-7, 61-62 & 73.90 & 83.19 & 1.00 & 30.83 \\
\hline U1385E-9H-7, 73-74 & 74.02 & 83.31 & 2.03 & 22.90 \\
\hline U1385E-9H-7, 85-86 & 74.14 & 83.43 & 0.88 & 31.56 \\
\hline U1385E-9H-7, 97-98 & 74.26 & 83.55 & 1.05 & 27.38 \\
\hline U1385D-10H-1, 1-2 & 79.91 & 83.73 & 1.10 & 33.06 \\
\hline U1385D-10H-1, 13-14 & 80.03 & 83.85 & 0.99 & 33.19 \\
\hline U1385D-10H-1, 25-26 & 80.15 & 83.97 & 0.94 & 20.03 \\
\hline U1385D-10H-1, 37-38 & 80.27 & 84.09 & 0.82 & 19.38 \\
\hline U1385D-10H-1, 49-50 & 80.39 & 84.21 & 0.75 & 21.12 \\
\hline U1385D-10H-1, 61-62 & 80.51 & 84.33 & 0.91 & 23.63 \\
\hline U1385D-10H-1, 73-74 & 80.63 & 84.45 & 1.08 & 23.98 \\
\hline U1385D-10H-1, 85-86 & 80.75 & 84.57 & 1.13 & 26.25 \\
\hline U1385D-10H-1, 97-98 & 80.87 & 84.69 & 1.25 & 25.90 \\
\hline
\end{tabular}

\begin{tabular}{|c|c|c|c|c|}
\hline $\begin{array}{l}\text { Hole, core, section, } \\
\text { interval }(\mathrm{cm})\end{array}$ & $\begin{array}{l}\text { Top depth } \\
\text { (mbsf) }\end{array}$ & $\begin{array}{l}\text { Top depth } \\
\text { (mcd) }\end{array}$ & $\begin{array}{l}\text { TOC } \\
\text { (wt\%) }\end{array}$ & $\begin{array}{l}\mathrm{CaCO}_{3} \\
(\mathrm{wt} \%)\end{array}$ \\
\hline U1385D-10H-1, 109-110 & 80.99 & 84.81 & 1.20 & 24.48 \\
\hline U1385D-10H-1, 121-122 & 81.11 & 84.93 & 1.29 & 25.88 \\
\hline U1385D-10H-1, 133-134 & 81.23 & 85.05 & 1.08 & 27.57 \\
\hline U1385D-10H-1, 145-146 & 81.35 & 85.17 & 1.20 & 26.29 \\
\hline U1385D-10H-2, 1-2 & 81.41 & 85.23 & 0.95 & 26.02 \\
\hline U1385D-10H-2, 13-14 & 81.53 & 85.35 & 1.14 & 26.39 \\
\hline U1385D-10H-2, 25-26 & 81.65 & 85.47 & 1.20 & 25.18 \\
\hline U1385D-10H-2, 37-38 & 81.77 & 85.59 & 1.39 & 28.92 \\
\hline U1385D-10H-2, 49-50 & 81.89 & 85.71 & 1.06 & 34.04 \\
\hline U1385D-10H-2, 61-62 & 82.01 & 85.83 & 1.07 & 28.86 \\
\hline U1385D-10H-2, 73-74 & 82.13 & 85.95 & 0.84 & 20.05 \\
\hline U1385D-10H-2, 85-86 & 82.25 & 86.07 & 1.17 & 25.06 \\
\hline U1385D-10H-2, 97-98 & 82.37 & 86.19 & 0.85 & 30.70 \\
\hline U1385D-10H-2, 109-110 & 82.49 & 86.31 & 1.07 & 24.38 \\
\hline U1385D-10H-2, 121-122 & 82.61 & 86.43 & 0.90 & 25.72 \\
\hline U1385D-10H-2, 133-134 & 82.73 & 86.55 & 1.20 & 26.10 \\
\hline U1385D-10H-2, 145-146 & 82.85 & 86.67 & 1.16 & 20.79 \\
\hline U1385D-10H-3, 1-2 & 82.91 & 86.73 & 1.24 & 20.60 \\
\hline U1385D-10H-3, 13-14 & 83.03 & 86.85 & 1.17 & 23.46 \\
\hline U1385D-10H-3, 25-26 & 83.15 & 86.97 & 0.67 & 25.80 \\
\hline U1385D-10H-3, 37-38 & 83.27 & 87.09 & 1.02 & 21.29 \\
\hline U1385D-10H-3, 49-50 & 83.39 & 87.21 & 0.83 & 20.51 \\
\hline U1385D-10H-3, 61-62 & 83.51 & 87.33 & 0.92 & 24.41 \\
\hline U1385D-10H-3, 73-74 & 83.63 & 87.45 & 1.04 & 23.50 \\
\hline U1385D-10H-3, 85-86 & 83.75 & 87.57 & 0.87 & 25.65 \\
\hline U1385D-10H-3, 97-98 & 83.87 & 87.69 & 1.00 & 23.08 \\
\hline U1385D-10H-3, 109-110 & 83.99 & 87.81 & 0.75 & 26.19 \\
\hline U1385D-10H-3, 121-122 & 84.11 & 87.93 & 1.13 & 24.20 \\
\hline U1385D-10H-3, 133-134 & 84.23 & 88.05 & 1.00 & 22.63 \\
\hline U1385D-10H-3, 145-146 & 84.35 & 88.17 & 1.03 & 20.14 \\
\hline U1385D-10H-4, 1-2 & 84.42 & 88.24 & 0.90 & 22.03 \\
\hline U1385D-10H-4, 13-14 & 84.54 & 88.36 & 0.95 & 23.34 \\
\hline U1385D-10H-4, 25-26 & 84.66 & 88.48 & 0.97 & 21.60 \\
\hline U1385D-10H-4, 37-38 & 84.78 & 88.60 & 1.06 & 22.53 \\
\hline U1385D-10H-4, 49-50 & 84.90 & 88.72 & 0.91 & 21.83 \\
\hline U1385D-10H-4, 61-62 & 85.02 & 88.84 & 1.17 & 22.87 \\
\hline U1385D-10H-4, 73-74 & 85.14 & 88.96 & 1.09 & 18.90 \\
\hline U1385D-10H-4, 85-86 & 85.26 & 89.08 & 1.00 & 21.40 \\
\hline U1385D-10H-4, 97-98 & 85.38 & 89.20 & 0.88 & 22.86 \\
\hline U1385D-10H-4, 109-110 & 85.50 & 89.32 & 0.50 & 19.95 \\
\hline U1385D-10H-4, 121-122 & 85.62 & 89.44 & 1.43 & 27.62 \\
\hline U1385D-10H-4, 133-134 & 85.74 & 89.56 & 1.26 & 24.45 \\
\hline U1385D-10H-4, 145-146 & 85.86 & 89.68 & 0.99 & 18.97 \\
\hline U1385D-10H-5, 1-2 & 85.95 & 89.77 & 0.87 & 18.43 \\
\hline U1385D-10H-5, 13-14 & 86.07 & 89.89 & 1.01 & 24.93 \\
\hline U1385D-10H-5, 25-26 & 86.19 & 90.01 & 1.00 & 25.72 \\
\hline U1385D-10H-5, 37-38 & 86.31 & 90.13 & 0.53 & 27.21 \\
\hline U1385D-10H-5, 49-50 & 86.43 & 90.25 & 0.88 & 23.80 \\
\hline U1385D-10H-5, 61-62 & 86.55 & 90.37 & 0.70 & 35.88 \\
\hline U1385D-10H-5, 73-74 & 86.67 & 90.49 & 0.97 & 37.66 \\
\hline U1385D-10H-5, 85-86 & 86.79 & 90.61 & 0.63 & 38.21 \\
\hline U1385D-10H-5, 97-98 & 86.91 & 90.73 & 0.96 & 35.93 \\
\hline U1385D-10H-5, 109-110 & 87.03 & 90.85 & 0.92 & 39.24 \\
\hline U1385D-10H-5, 121-122 & 87.15 & 90.97 & 0.89 & 39.34 \\
\hline U1385D-10H-5, 133-134 & 87.27 & 91.09 & 0.83 & 40.50 \\
\hline U1385D-10H-5, 145-146 & 87.39 & 91.21 & 0.87 & 40.86 \\
\hline U1385D-10H-6, 1-2 & 87.46 & 91.28 & 0.64 & 44.71 \\
\hline U1385D-10H-6, 13-14 & 87.58 & 91.40 & 0.94 & 40.51 \\
\hline U1385D-10H-6, 25-26 & 87.70 & 91.52 & 1.08 & 42.21 \\
\hline U1385D-10H-6, 37-38 & 87.82 & 91.64 & 0.93 & 37.85 \\
\hline U1385D-10H-6, 49-50 & 87.94 & 91.76 & 0.84 & 32.93 \\
\hline U1385D-10H-6, 61-62 & 88.06 & 91.88 & 0.84 & 25.82 \\
\hline U1385D-10H-6, 73-74 & 88.18 & 92.00 & 0.72 & 20.98 \\
\hline U1385D-10H-6, 85-86 & 88.30 & 92.12 & 0.71 & 28.71 \\
\hline U1385D-10H-6, 97-98 & 88.42 & 92.24 & 0.76 & 32.72 \\
\hline U1385D-10H-6, 109-110 & 88.54 & 92.36 & 0.81 & 32.81 \\
\hline U1385D-10H-6, 121 & 88.66 & 92.48 & 0.94 & 32.12 \\
\hline
\end{tabular}


Table T1 (continued).

\begin{tabular}{|c|c|c|c|c|}
\hline $\begin{array}{l}\text { Hole, core, section, } \\
\text { interval }(\mathrm{cm})\end{array}$ & $\begin{array}{l}\text { Top depth } \\
\text { (mbsf) }\end{array}$ & $\begin{array}{l}\text { Top depth } \\
\text { (mcd) }\end{array}$ & $\begin{array}{c}\text { TOC } \\
\text { (wt\%) }\end{array}$ & $\begin{array}{l}\mathrm{CaCO}_{3} \\
(\mathrm{wt} \%)\end{array}$ \\
\hline U1385D-10H-6, 133-134 & 88.78 & 92.60 & 0.81 & 35.67 \\
\hline U1385D-10H-6, 145-146 & 88.90 & 92.72 & 1.13 & 29.20 \\
\hline U1385D-10H-7, 1-2 & 88.97 & 92.79 & 0.77 & 34.19 \\
\hline U1385D-10H-7, 13-14 & 89.09 & 92.91 & 2.22 & 20.57 \\
\hline U1385D-10H-7, 25-26 & 89.21 & 93.03 & 0.85 & 31.93 \\
\hline U1385D-10H-7, 37-38 & 89.33 & 93.15 & 2.72 & 12.12 \\
\hline U1385D-10H-7, 49-50 & 89.45 & 93.27 & 0.90 & 33.09 \\
\hline U1385E-11H-1, 1-2 & 82.51 & 93.43 & 1.23 & 31.89 \\
\hline U1385E-11H-1, 13-14 & 82.63 & 93.55 & 0.85 & 34.02 \\
\hline U1385E-11H-1, 25-26 & 82.75 & 93.67 & 0.77 & 34.58 \\
\hline U1385E-11H-1, 37-38 & 82.87 & 93.79 & 1.13 & 28.92 \\
\hline U1385E-11H-1, 49-50 & 82.99 & 93.91 & 1.08 & 32.32 \\
\hline U1385E-11H-1, 61-62 & 83.11 & 94.03 & 1.16 & 29.79 \\
\hline U1385E-11H-1, 73-74 & 83.23 & 94.15 & 0.75 & 29.37 \\
\hline U1385E-11H-1, 85-86 & 83.35 & 94.27 & 0.64 & 26.73 \\
\hline U1385E-11H-1, 97-98 & 83.47 & 94.39 & 0.84 & 30.37 \\
\hline U1385E-11H-1, 109-110 & 83.59 & 94.51 & 0.94 & 34.61 \\
\hline U1385E-11H-1, 121-122 & 83.71 & 94.63 & 0.64 & 22.83 \\
\hline U1385E-11H-1, 133-134 & 83.83 & 94.75 & 1.08 & 33.61 \\
\hline U1385E-11H-1, 145-146 & 83.95 & 94.87 & 0.79 & 33.99 \\
\hline U1385E-11H-2, 1-2 & 84.01 & 94.93 & 0.62 & 40.41 \\
\hline U1385E-11H-2, 13-14 & 84.13 & 95.05 & 0.81 & 43.19 \\
\hline U1385E-11H-2, 25-26 & 84.25 & 95.17 & 0.70 & 45.64 \\
\hline U1385E-11H-2, 37-38 & 84.37 & 95.29 & 0.77 & 38.88 \\
\hline U1385E-11H-2, 49-50 & 84.49 & 95.41 & 0.81 & 40.42 \\
\hline U1385E-11H-2, 61-62 & 84.61 & 95.53 & 0.67 & 41.84 \\
\hline U1385E-11H-2, 73-74 & 84.73 & 95.65 & 0.88 & 38.06 \\
\hline U1385E-11H-2, 85-86 & 84.85 & 95.77 & 1.12 & 26.50 \\
\hline U1385E-11H-2, 97-98 & 84.97 & 95.89 & 0.70 & 24.00 \\
\hline U1385E-11H-2, 109-110 & 85.09 & 96.01 & 0.82 & 18.17 \\
\hline U1385E-11H-2, 121-122 & 85.21 & 96.13 & 0.94 & 19.89 \\
\hline
\end{tabular}

\begin{tabular}{lcccc}
\hline $\begin{array}{c}\text { Hole, core, section, } \\
\text { interval }(\mathrm{cm})\end{array}$ & $\begin{array}{c}\text { Top depth } \\
\text { (mbsf) }\end{array}$ & $\begin{array}{c}\text { Top depth } \\
\text { (mcd) }\end{array}$ & $\begin{array}{c}\text { TOC } \\
\text { (wt\%) }\end{array}$ & $\begin{array}{c}\mathrm{CaCO}_{3} \\
\text { (wt\%) }\end{array}$ \\
\hline U1385E-11H-2, 133-134 & 85.33 & 96.25 & 1.29 & 26.77 \\
U1385E-11H-2, 145-146 & 85.45 & 96.37 & 0.99 & 33.97 \\
U1385E-11H-3, 1-2 & 85.52 & 96.44 & 1.09 & 34.98 \\
U1385E-11H-3, 13-14 & 85.64 & 96.56 & 1.13 & 29.00 \\
U1385E-11H-3, 25-26 & 85.76 & 96.68 & 0.94 & 30.54 \\
U1385E-11H-3, 37-38 & 85.88 & 96.80 & 1.21 & 29.60 \\
U1385E-11H-3, 49-50 & 86.00 & 96.92 & 1.10 & 25.69 \\
U1385E-11H-3, 61-62 & 86.12 & 97.04 & 0.94 & 26.12 \\
U1385E-11H-3, 73-74 & 86.24 & 97.16 & 0.97 & 31.61 \\
U1385E-11H-3, 85-86 & 86.36 & 97.28 & 0.90 & 30.48 \\
U1385E-11H-3,97-98 & 86.48 & 97.40 & 0.83 & 30.15 \\
U1385E-11H-3, 121-122 & 86.72 & 97.64 & 1.07 & 36.38 \\
U1385E-11H-3, 133-134 & 86.84 & 97.76 & 0.98 & 44.26 \\
U1385E-11H-3, 145-146 & 86.96 & 97.88 & 0.86 & 40.86 \\
U1385E-11H-4, 1-2 & 87.02 & 97.94 & 0.94 & 34.14 \\
U1385E-11H-4, 13-14 & 87.14 & 98.06 & 0.97 & 30.55 \\
U1385E-11H-4, 25-26 & 87.26 & 98.18 & 0.68 & 23.82 \\
U1385E-11H-4, 37-38 & 87.38 & 98.30 & 0.83 & 30.97 \\
U1385E-11H-4, 49-50 & 87.50 & 98.42 & 0.64 & 37.44 \\
U1385E-11H-4, 61-62 & 87.62 & 98.54 & 0.79 & 36.03 \\
U1385E-11H-4, 85-86 & 87.86 & 98.78 & 0.69 & 35.27 \\
U1385E-11H-4, 97-98 & 87.98 & 98.90 & 0.65 & 35.26 \\
U1385E-11H-4, 109-110 & 88.10 & 99.02 & 0.60 & 33.81 \\
U1385E-11H-4, 121-122 & 88.22 & 99.14 & 0.75 & 32.28 \\
U1385E-11H-4, 133-134 & 88.34 & 99.26 & 0.69 & 35.37 \\
U1385E-11H-4, 145-146 & 88.46 & 99.38 & 0.89 & 29.55 \\
U1385E-11H-5, 1-2 & 88.53 & 99.45 & 1.13 & 30.15 \\
U1385E-11H-5, 13-14 & 88.65 & 99.57 & 1.36 & 26.07 \\
U1385E-11H-5, 25-26 & 88.77 & 99.69 & 1.24 & 25.08 \\
U1385E-11H-5, 37-38 & 88.89 & 99.81 & 1.11 & 22.83 \\
U1385E-11H-5, 49-50 & 89.01 & 99.93 & 0.98 & 19.18 \\
\hline
\end{tabular}


Table T2. Calcium carbonate and total organic carbon (TOC) concentrations from 160 to 220 mcd, Site U1391. (Continued on next page.)

\begin{tabular}{|c|c|c|c|c|}
\hline $\begin{array}{l}\text { Hole, core, section, } \\
\text { interval }(\mathrm{cm})\end{array}$ & $\begin{array}{l}\text { Top depth } \\
\text { (mbsf) }\end{array}$ & $\begin{array}{l}\text { Top depth } \\
\text { (mcd) }\end{array}$ & $\begin{array}{l}\text { TOC } \\
\text { (wt\%) }\end{array}$ & $\begin{array}{l}\mathrm{CaCO}_{3} \\
(\mathrm{wt} \%)\end{array}$ \\
\hline \multicolumn{5}{|l|}{ 339- } \\
\hline U1391A-11H-2, 120-121 & 92.21 & 100.02 & 1.18 & 23.90 \\
\hline U1391A-11H-3, 30-31 & 92.68 & 100.49 & 1.33 & 20.76 \\
\hline U1391A-11H-3, 75-76 & 93.13 & 100.94 & 1.34 & 20.11 \\
\hline U1391A-11H-4, 0-1 & 93.68 & 101.49 & 1.61 & 17.00 \\
\hline U1391A-11H-4, 45-46 & 94.13 & 101.94 & 1.29 & 19.79 \\
\hline U1391A-11H-4, 105-106 & 94.73 & 102.54 & 0.41 & 29.59 \\
\hline U1391A-11H-5, 15-16 & 95.28 & 103.09 & 0.62 & 37.00 \\
\hline U1391A-11H-5, 60-61 & 95.73 & 103.54 & 0.78 & 36.26 \\
\hline U1391B-11H-1, 120-121 & 96.20 & 103.85 & 1.62 & 19.70 \\
\hline U1391B-11H-2, 30-31 & 96.71 & 104.36 & 0.68 & 26.95 \\
\hline U1391B-11H-2, 90-91 & 97.31 & 104.96 & 0.62 & 26.42 \\
\hline U1391B-11H-3, 15-16 & 97.98 & 105.63 & 0.67 & 25.79 \\
\hline U1391B-11H-3, 75-76 & 98.58 & 106.23 & 0.75 & 27.43 \\
\hline U1391B-11H-3, 135-136 & 99.18 & 106.83 & 0.96 & 19.04 \\
\hline U1391B-11H-4, 45-46 & 99.69 & 107.34 & 0.68 & 29.45 \\
\hline U1391B-11H-4, 105-106 & 100.29 & 107.94 & 0.81 & 21.75 \\
\hline U1391B-11H-5, 15-16 & 100.81 & 108.46 & 0.78 & 22.76 \\
\hline U1391A-12H-2, 60-61 & 100.29 & 109.01 & 0.59 & 37.62 \\
\hline U1391B-11H-5, 75-76 & 101.41 & 109.06 & 0.94 & 31.76 \\
\hline U1391A-12H-2, 105-106 & 100.74 & 109.46 & 1.08 & 32.78 \\
\hline U1391A-12H-3, 15-16 & 101.25 & 109.97 & 0.83 & 19.82 \\
\hline U1391A-12H-3, 75-76 & 101.85 & 110.57 & 1.10 & 41.05 \\
\hline U1391A-12H-3, 120-121 & 102.30 & 111.02 & 0.83 & 39.74 \\
\hline U1391A-12H-4, 30-31 & 102.73 & 111.45 & 1.04 & 33.70 \\
\hline U1391A-12H-4, 75-76 & 103.18 & 111.90 & 0.85 & 22.73 \\
\hline U1391A-12H-4, 135-136 & 103.78 & 112.50 & 1.10 & 23.58 \\
\hline U1391A-12H-5, 45-46 & 104.26 & 112.98 & 1.16 & 34.64 \\
\hline U1391A-12H-5, 105-106 & 104.86 & 113.58 & 1.00 & 27.54 \\
\hline U1391A-12H-6, 30-31 & 105.37 & 114.09 & 0.76 & 22.81 \\
\hline U1391A-12H-6, 75-76 & 105.82 & 114.54 & 0.90 & 19.71 \\
\hline U1391A-12H-6, 105-106 & 106.12 & 114.84 & 1.20 & 34.59 \\
\hline U1391B-12H-2, 15-16 & 106.07 & 115.16 & 1.44 & 32.93 \\
\hline U1391B-12H-2, 75-76 & 106.67 & 115.76 & 1.30 & 37.42 \\
\hline U1391B-12H-2, 135-136 & 107.27 & 116.36 & 1.12 & 38.16 \\
\hline U1391B-12H-3, 45-46 & 107.78 & 116.87 & 1.16 & 36.68 \\
\hline U1391B-12H-3, 105-106 & 108.38 & 117.47 & 1.35 & 34.07 \\
\hline U1391B-12H-4, 15-16 & 108.89 & 117.98 & 1.29 & 35.39 \\
\hline U1391B-12H-4, 75-76 & 109.49 & 118.58 & 1.22 & 36.34 \\
\hline U1391B-12H-4, 135-136 & 110.09 & 119.18 & 1.36 & 35.66 \\
\hline U1391B-12H-5, 45-46 & 110.61 & 119.70 & 1.22 & 36.35 \\
\hline U1391A-13H-2, 75-76 & 110.49 & 120.24 & 1.10 & 37.23 \\
\hline U1391B-12H-5, 105-106 & 111.21 & 120.30 & 0.95 & 44.06 \\
\hline U1391A-13H-2, 105-106 & 110.79 & 120.54 & 1.01 & 37.46 \\
\hline U1391A-13H-3, 15-16 & 111.30 & 121.05 & 1.00 & 36.25 \\
\hline U1391A-13H-3, 60-61 & 111.75 & 121.50 & 1.21 & 36.77 \\
\hline U1391A-13H-3, 105-106 & 112.20 & 121.95 & 1.10 & 39.64 \\
\hline U1391A-13H-4, 30-31 & 112.81 & 122.56 & 1.05 & 37.99 \\
\hline U1391A-13H-4, 75-76 & 113.26 & 123.01 & 1.05 & 41.09 \\
\hline U1391A-13H-4, 120-121 & 113.71 & 123.46 & 1.05 & 33.02 \\
\hline U1391A-13H-5, 45-46 & 114.27 & 124.02 & 1.57 & 26.17 \\
\hline U1391A-13H-5, 90-91 & 114.72 & 124.47 & 1.61 & 32.59 \\
\hline U1391A-13H-6, 0-1 & 115.20 & 124.95 & 0.60 & 29.75 \\
\hline U1391A-13H-6, 60-61 & 115.80 & 125.55 & 0.78 & 17.41 \\
\hline U1391A-13H-6, 105-106 & 116.25 & 126.00 & 0.77 & 23.33 \\
\hline U1391B-13H-3, 90-91 & 117.72 & 127.19 & 1.18 & 33.01 \\
\hline U1391B-13H-4, 0-1 & 118.23 & 127.70 & 1.18 & 31.35 \\
\hline U1391B-13H-4, 60-61 & 118.83 & 128.30 & 0.89 & 28.70 \\
\hline U1391B-13H-4, 120-121 & 119.43 & 128.90 & 1.19 & 32.71 \\
\hline U1391B-13H-5, 30-31 & 119.94 & 129.41 & 1.16 & 36.56 \\
\hline U1391B-13H-5, 90-91 & 120.54 & 130.01 & 0.94 & 37.79 \\
\hline U1391B-13Н-6, 0-1 & 121.07 & 130.54 & 1.11 & 34.57 \\
\hline U1391A-14H-1, 119-120 & 119.29 & 130.90 & 1.08 & 33.99 \\
\hline U1391A-14H-2, 30-31 & 119.83 & 131.44 & 1.12 & 30.91 \\
\hline U1391A-14H-2, 75-76 & 120.28 & 131.89 & 1.29 & 34.03 \\
\hline U1391A-14H-2, 135-136 & 120.88 & 132.49 & 1.17 & 39.55 \\
\hline U1391A-14H-3, 46-47 & 121.40 & 133.01 & 0.84 & 18.76 \\
\hline
\end{tabular}

\begin{tabular}{|c|c|c|c|c|}
\hline $\begin{array}{l}\text { Hole, core, section, } \\
\text { interval }(\mathrm{cm})\end{array}$ & $\begin{array}{l}\text { Top depth } \\
\text { (mbsf) }\end{array}$ & $\begin{array}{l}\text { Top depth } \\
\text { (mcd) }\end{array}$ & $\begin{array}{l}\text { TOC } \\
\text { (wt\%) }\end{array}$ & $\begin{array}{l}\mathrm{CaCO}_{3} \\
\text { (wt\%) }\end{array}$ \\
\hline U1391A-14H-3, 90-91 & 121.84 & 133.45 & 1.72 & 15.26 \\
\hline U1391A-14H-4, 2-3 & 122.42 & 134.03 & 1.26 & 26.73 \\
\hline U1391A-14H-4, 46-47 & 122.86 & 134.47 & 1.10 & 21.55 \\
\hline U1391A-14H-4, 106-107 & 123.46 & 135.07 & 1.37 & 30.00 \\
\hline U1391A-14H-5, 15-16 & 123.95 & 135.56 & 1.16 & 30.30 \\
\hline U1391A-14H-5, 75-76 & 124.55 & 136.16 & 1.08 & 36.34 \\
\hline U1391B-14H-1, 59-60 & 124.09 & 136.56 & 1.03 & 35.21 \\
\hline U1391A-14H-5, 119-120 & 124.99 & 136.60 & 1.21 & 37.25 \\
\hline U1391B-14H-1, 118-119 & 124.68 & 137.15 & 1.13 & 39.73 \\
\hline U1391B-14H-2, 30-31 & 125.22 & 137.69 & 1.20 & 40.03 \\
\hline U1391B-14H-2, 90-91 & 125.82 & 138.29 & 1.09 & 39.24 \\
\hline U1391B-14H-3, 2-3 & 126.36 & 138.83 & 1.05 & 38.59 \\
\hline U1391B-14H-3, 59-60 & 126.93 & 139.40 & 1.11 & 38.96 \\
\hline U1391B-14H-3, 119-120 & 127.53 & 140.00 & 1.12 & 32.79 \\
\hline U1391B-14H-4, 30-31 & 128.06 & 140.53 & 1.08 & 26.49 \\
\hline U1391B-14H-4, 90-91 & 128.66 & 141.13 & 0.99 & 29.13 \\
\hline U1391B-14H-5, 2-3 & 129.19 & 141.66 & 1.12 & 40.14 \\
\hline U1391B-14H-5, 59-60 & 129.76 & 142.23 & 1.09 & 32.95 \\
\hline U1391B-14H-5, 119-120 & 130.36 & 142.83 & 1.08 & 26.55 \\
\hline U1391B-14H-6, 30-31 & 130.92 & 143.39 & 0.79 & 21.78 \\
\hline U1391B-14H-6, 90-91 & 131.52 & 143.99 & 0.76 & 24.25 \\
\hline U1391A-15H-1, 90-91 & 128.50 & 144.04 & 0.99 & 22.04 \\
\hline U1391A-15H-1, 135-136 & 128.95 & 144.49 & 1.33 & 17.31 \\
\hline U1391A-15H-2, 46-47 & 129.51 & 145.05 & 0.94 & 17.62 \\
\hline U1391A-15H-2, 90-91 & 129.95 & 145.49 & 0.78 & 23.43 \\
\hline U1391A-15H-2, 135-136 & 130.40 & 145.94 & 0.86 & 32.29 \\
\hline U1391A-15H-3, 46-47 & 130.89 & 146.43 & 1.23 & 33.96 \\
\hline U1391A-15H-3, 106-107 & 131.49 & 147.03 & 0.82 & 23.55 \\
\hline U1391A-15H-4, 15-16 & 131.98 & 147.52 & 2.10 & 23.37 \\
\hline U1391A-15H-4, 59-60 & 132.42 & 147.96 & 0.61 & 31.86 \\
\hline U1391A-15H-4, 106-107 & 132.89 & 148.43 & 0.68 & 24.75 \\
\hline U1391A-15H-5, 15-16 & 133.41 & 148.95 & 0.78 & 24.70 \\
\hline U1391A-15H-5, 75-76 & 134.01 & 149.55 & 0.69 & 20.38 \\
\hline U1391A-15H-5, 135-136 & 134.61 & 150.15 & 0.75 & 19.00 \\
\hline U1391A-15H-6, 30-31 & 135.03 & 150.57 & 0.92 & 21.46 \\
\hline U1391B-15H-2, 119-120 & 134.62 & 150.88 & 1.09 & 29.37 \\
\hline U1391B-15H-3, 30-31 & 135.10 & 151.36 & 0.99 & 24.91 \\
\hline U1391B-15H-3, 90-91 & 135.70 & 151.96 & 0.76 & 19.18 \\
\hline U1391B-15H-4, 0-1 & 136.21 & 152.47 & 1.14 & 16.26 \\
\hline U1391B-15H-4, 59-60 & 136.80 & 153.06 & 0.99 & 26.92 \\
\hline U1391B-15H-4, 120-121 & 137.41 & 153.67 & 1.22 & 27.09 \\
\hline U1391B-15H-5, 30-31 & 138.01 & 154.27 & 0.88 & 27.72 \\
\hline U1391B-15H-5, 90-91 & 138.61 & 154.87 & 0.82 & 29.37 \\
\hline U1391B-15H-6, 2-3 & 139.20 & 155.46 & 1.02 & 27.93 \\
\hline U1391B-15H-6, 60-61 & 139.78 & 156.04 & 1.05 & 25.98 \\
\hline U1391B-15H-6, 120-121 & 140.38 & 156.64 & 1.71 & 18.62 \\
\hline U1391B-15H-7, 30-31 & 140.94 & 157.20 & 0.96 & 26.06 \\
\hline U1391B-15H-7, 89-90 & 141.53 & 157.79 & 0.79 & 27.54 \\
\hline U1391A-16H-2, 32-33 & 137.85 & 158.09 & 1.15 & 24.54 \\
\hline U1391A-16H-2, 75-76 & 138.28 & 158.52 & 0.90 & 27.60 \\
\hline U1391A-16H-2, 132-133 & 138.85 & 159.09 & 1.29 & 26.77 \\
\hline U1391A-16H-3, 30-31 & 139.25 & 159.49 & 1.13 & 17.78 \\
\hline U1391A-16H-3, 90-91 & 139.85 & 160.09 & 0.86 & 19.55 \\
\hline U1391A-16H-3, 135-136 & 140.30 & 160.54 & 1.08 & 17.44 \\
\hline U1391A-16H-4, 31-32 & 140.70 & 160.94 & 1.13 & 14.35 \\
\hline U1391A-16H-4, 62-63 & 141.01 & 161.25 & 0.99 & 15.26 \\
\hline U1391A-16H-4, 90-91 & 141.29 & 161.53 & 0.86 & 18.73 \\
\hline U1391A-16H-5, 0-1 & 141.86 & 162.10 & 1.31 & 16.45 \\
\hline U1391A-16H-5, 59-60 & 142.45 & 162.69 & 0.88 & 22.77 \\
\hline U1391A-16H-5, 105-106 & 142.91 & 163.15 & 0.85 & 22.68 \\
\hline U1391A-16H-6, 0-1 & 143.23 & 163.47 & 1.26 & 32.78 \\
\hline U1391A-16H-6, 30-31 & 143.53 & 163.77 & 0.83 & 35.36 \\
\hline U1391A-16H-6, 90-91 & 144.13 & 164.37 & 0.93 & 26.06 \\
\hline U1391A-16H-6, 150-151 & 144.73 & 164.97 & 1.19 & 23.58 \\
\hline U1391A-16H-7, 30-31 & 145.06 & 165.30 & 0.87 & 28.59 \\
\hline U1391A-16H-7, 88-89 & 145.64 & 165.88 & 1.00 & 34.79 \\
\hline U1391B-16H-2, 15-16 & 144.07 & 166.08 & 1.01 & 35.97 \\
\hline
\end{tabular}


Table T2 (continued).

\begin{tabular}{|c|c|c|c|c|}
\hline $\begin{array}{l}\text { Hole, core, section, } \\
\text { interval }(\mathrm{cm})\end{array}$ & $\begin{array}{l}\text { Top depth } \\
\text { (mbsf) }\end{array}$ & $\begin{array}{l}\text { Top depth } \\
\text { (mcd) }\end{array}$ & $\begin{array}{l}\text { TOC } \\
\text { (wt\%) }\end{array}$ & $\begin{array}{l}\mathrm{CaCO}_{3} \\
\text { (wt\%) }\end{array}$ \\
\hline U1391A-16H-7, 118-119 & 145.94 & 166.18 & 0.79 & 23.81 \\
\hline U1391B-16H-2, 75-76 & 144.67 & 166.68 & 0.67 & 17.82 \\
\hline U1391B-16H-2, 135-136 & 145.27 & 167.28 & 0.94 & 24.25 \\
\hline U1391B-16H-3, 60-61 & 145.94 & 167.95 & 0.97 & 27.99 \\
\hline U1391B-16H-3, 120-121 & 146.54 & 168.55 & 0.97 & 27.10 \\
\hline U1391B-16H-4, 30-31 & 147.15 & 169.16 & 0.95 & 27.53 \\
\hline U1391B-16H-4, 89-90 & 147.74 & 169.75 & 1.01 & 28.33 \\
\hline U1391B-16H-5, 0-1 & 148.32 & 170.33 & 0.97 & 28.20 \\
\hline U1391B-16H-5, 59-60 & 148.91 & 170.92 & 0.78 & 27.22 \\
\hline U1391B-16H-5, 120-121 & 149.52 & 171.53 & 0.94 & 26.05 \\
\hline U1391A-17H-2, 75-76 & 148.75 & 172.48 & 1.65 & 21.24 \\
\hline U1391A-17H-2, 135-136 & 149.35 & 173.08 & 1.03 & 23.02 \\
\hline U1391A-17H-3, 45-46 & 149.84 & 173.57 & 1.15 & 18.46 \\
\hline U1391A-17H-3, 105-106 & 150.44 & 174.17 & 0.76 & 25.23 \\
\hline U1391A-17H-4, 15-16 & 150.96 & 174.69 & 0.87 & 28.29 \\
\hline U1391A-17H-4, 75-76 & 151.56 & 175.29 & 1.01 & 25.73 \\
\hline U1391A-17H-4, 135-136 & 152.16 & 175.89 & 0.94 & 21.96 \\
\hline U1391A-17H-5, 45-46 & 152.69 & 176.42 & 0.86 & 20.99 \\
\hline U1391B-17H-1, 90-91 & 152.9 & 176.71 & 0.81 & 31.17 \\
\hline U1391B-17H-2, 0-1 & 153.39 & 177.20 & 0.90 & 28.69 \\
\hline U1391B-17H-2, 60-61 & 153.99 & 177.80 & 0.74 & 30.04 \\
\hline U1391B-17H-3, 45-46 & 155.24 & 179.05 & 0.90 & 20.25 \\
\hline U1391B-17H-3, 103-104 & 155.82 & 179.63 & 1.15 & 24.93 \\
\hline U1391B-17H-4, 15-16 & 156.40 & 180.21 & 0.81 & 21.35 \\
\hline U1391B-17H-4, 75-76 & 157.00 & 180.81 & 0.87 & 23.35 \\
\hline U1391B-17H-4, 135-136 & 157.60 & 181.41 & 0.79 & 22.37 \\
\hline U1391B-17H-5, 45-46 & 158.20 & 182.01 & 0.90 & 19.96 \\
\hline U1391A-18H-2, 45-46 & 157.58 & 182.31 & 1.21 & 24.96 \\
\hline U1391A-18H-2, 103-104 & 158.16 & 182.89 & 0.99 & 32.64 \\
\hline U1391A-18H-3, 30-31 & 158.82 & 183.55 & 1.30 & 18.68 \\
\hline U1391A-18H-3, 90-91 & 159.42 & 184.15 & 1.16 & 32.15 \\
\hline U1391A-18H-4, 15-16 & 159.88 & 184.61 & 1.06 & 28.44 \\
\hline U1391A-18H-4, 75-76 & 160.48 & 185.21 & 0.92 & 29.83 \\
\hline U1391A-18H-5, 0-1 & 161.15 & 185.88 & 0.89 & 29.75 \\
\hline U1391A-18H-5, 66-67 & 161.81 & 186.54 & 1.13 & 33.69 \\
\hline U1391A-18H-5, 120-121 & 162.35 & 187.08 & 1.02 & 24.85 \\
\hline U1391A-18H-6, 29-30 & 162.87 & 187.60 & 0.69 & 31.53 \\
\hline U1391A-18H-6, 90-91 & 163.48 & 188.21 & 0.89 & 31.57 \\
\hline U1391B-18H-2, 2-3 & 162.93 & 188.35 & 1.14 & 31.31 \\
\hline U1391B-18H-2, 60-61 & 163.51 & 188.93 & 0.93 & 34.83 \\
\hline U1391B-18H-2, 122-123 & 164.13 & 189.55 & 0.75 & 34.41 \\
\hline U1391B-18H-3, 45-46 & 164.65 & 190.07 & 0.87 & 32.48 \\
\hline U1391B-18H-3, 105-106 & 165.25 & 190.67 & 1.12 & 30.13 \\
\hline U1391B-18H-4, 30-31 & 165.84 & 191.26 & 1.19 & 25.06 \\
\hline U1391B-18H-4, 90-91 & 166.44 & 191.86 & 1.12 & 21.30 \\
\hline U1391A-19H-1, 122-123 & 166.82 & 192.55 & 1.16 & 19.16 \\
\hline U1391A-19H-2, 30-31 & 167.21 & 192.94 & 1.18 & 19.53 \\
\hline
\end{tabular}

\begin{tabular}{|c|c|c|c|c|}
\hline $\begin{array}{l}\text { Hole, core, section, } \\
\text { interval }(\mathrm{cm})\end{array}$ & $\begin{array}{l}\text { Top depth } \\
\text { (mbsf) }\end{array}$ & $\begin{array}{l}\text { Top depth } \\
\text { (mcd) }\end{array}$ & $\begin{array}{l}\text { TOC } \\
\text { (wt\%) }\end{array}$ & $\begin{array}{l}\mathrm{CaCO}_{3} \\
\text { (wt\%) }\end{array}$ \\
\hline U1391A-19H-2, 92-93 & 167.83 & 193.56 & 1.15 & 19.20 \\
\hline U1391A-19H-3, 0-1 & 168.28 & 194.01 & 0.92 & 20.61 \\
\hline U1391A-19H-3, 60-61 & 168.88 & 194.61 & 1.11 & 17.23 \\
\hline U1391A-19H-3, 120-121 & 169.48 & 195.21 & 1.11 & 19.71 \\
\hline U1391A-19H-4, 30-31 & 170.05 & 195.78 & 1.29 & 17.25 \\
\hline U1391A-19H-4, 92-93 & 170.67 & 196.40 & 0.93 & 20.16 \\
\hline U1391A-20X-1, 15-16 & 171.25 & 196.98 & 1.05 & 17.65 \\
\hline U1391A-20X-1, 75-76 & 171.85 & 197.58 & 0.85 & 19.97 \\
\hline U1391A-20X-1, 135-136 & 172.45 & 198.18 & 1.07 & 26.75 \\
\hline U1391A-20X-2, 45-46 & 173.05 & 198.78 & 1.13 & 28.79 \\
\hline U1391A-20X-2, 105-106 & 173.65 & 199.38 & 1.15 & 27.29 \\
\hline U1391A-20X-3, 15-16 & 174.25 & 199.98 & 1.04 & 26.38 \\
\hline U1391A-20X-3, 75-76 & 174.85 & 200.58 & 1.39 & 30.07 \\
\hline U1391A-20X-3, 135-136 & 175.45 & 201.18 & 1.00 & 19.11 \\
\hline U1391A-20X-4, 45-46 & 176.05 & 201.78 & 1.20 & 19.84 \\
\hline U1391A-20X-4, 105-106 & 176.65 & 202.38 & 1.09 & 29.26 \\
\hline U1391B-20X-2, 60-61 & 176.56 & 202.59 & 1.24 & 22.97 \\
\hline U1391B-20X-2, 120-121 & 177.16 & 203.19 & 0.78 & 22.24 \\
\hline U1391B-20X-3, 30-31 & 177.76 & 203.79 & 1.24 & 22.48 \\
\hline U1391B-20X-3, 92-93 & 178.38 & 204.41 & 0.86 & 23.56 \\
\hline U1391B-20X-4, 0-1 & 178.96 & 204.99 & 1.20 & 19.36 \\
\hline U1391B-20X-4, 60-61 & 179.56 & 205.59 & 0.69 & 23.75 \\
\hline U1391B-20X-4, 120-121 & 180.16 & 206.19 & 0.71 & 29.95 \\
\hline U1391A-21X-1, 45-46 & 181.15 & 207.13 & 1.32 & 24.33 \\
\hline U1391A-21X-1, 105-106 & 181.75 & 207.73 & 0.84 & 17.37 \\
\hline U1391A-21X-2, 15-16 & 182.35 & 208.33 & 0.74 & 18.88 \\
\hline U1391A-21X-2, 75-76 & 182.95 & 208.93 & 0.75 & 20.64 \\
\hline U1391A-21X-2, 135-136 & 183.55 & 209.53 & 1.12 & 15.46 \\
\hline U1391A-21X-3, 45-46 & 184.15 & 210.13 & 0.81 & 15.79 \\
\hline U1391A-21X-3, 105-106 & 184.75 & 210.73 & 0.89 & 16.20 \\
\hline U1391A-21X-4, 15-16 & 185.35 & 211.33 & 1.21 & 24.74 \\
\hline U1391A-21X-4, 75-76 & 185.95 & 211.93 & 1.22 & 22.34 \\
\hline U1391A-21X-4, 130-132 & 186.50 & 212.48 & 0.92 & 22.69 \\
\hline U1391A-21X-5, 45-46 & 187.08 & 213.06 & 0.96 & 31.32 \\
\hline U1391B-21X-1, 92-93 & 186.02 & 213.64 & 1.16 & 34.02 \\
\hline U1391B-21X-2, 15-16 & 186.51 & 214.13 & 1.07 & 33.83 \\
\hline U1391B-21X-2, 75-76 & 187.11 & 214.73 & 0.78 & 35.79 \\
\hline U1391B-21X-2, 135-136 & 187.71 & 215.33 & 0.92 & 34.88 \\
\hline U1391B-21X-3, 45-46 & 188.31 & 215.93 & 1.12 & 32.64 \\
\hline U1391B-21X-3, 105-106 & 188.91 & 216.53 & 0.97 & 27.98 \\
\hline U1391B-21X-4, 15-16 & 189.51 & 217.13 & 1.05 & 22.11 \\
\hline U1391B-21X-4, 75-76 & 190.11 & 217.73 & 0.91 & 22.30 \\
\hline U1391A-22X-2, 120-121 & 191.95 & 217.92 & 1.16 & 18.94 \\
\hline U1391A-22X-3, 30-31 & 192.55 & 218.52 & 1.04 & 19.40 \\
\hline U1391A-22X-3, 92-93 & 193.17 & 219.14 & 1.07 & 18.25 \\
\hline U1391A-22X-4, 0-1 & 193.75 & 219.72 & 1.03 & 17.06 \\
\hline
\end{tabular}

